\title{
IPEX syndrome with membrano-proliferative nephrotic syndrome
}

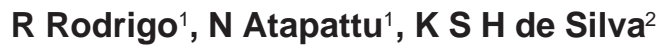

Ceylon Medical Journal 2013; 58: 43-44

\section{Introduction}

Immune dysregulation, polyendocrinopathy, enteropathy, $\mathrm{X}$-linked (IPEX) syndrome is a rare immune dysregulatory condition which is infrequently associated with nephrotic syndrome.

\section{Case report}

A two-month old male infant initially presented with cutaneous candidiasis. He is the second child of nonconsanguineous healthy parents whose first born is a bright six year old school girl. This boy was born at term following an uncomplicated antenatal period with a birth weight of $2.9 \mathrm{~kg}$. He was exclusively breast fed for six months. Initial tests for immunodeficiency including full blood count, HIV screening, CD 3,4,8,19,16 subsets, serum IgG, IgM and IgA levels were normal. IgE levels were not assessed due to non-availability of facilities.

At seven months of age he developed atopic dermatitis and alopecia. Pyrexia of unknown origin at the time prompted a trial of treatment for tuberculosis. Bone marrow, lymph node and skin biopsies were unremarkable. Over the next two months he had episodic non-bloody loose stools which grew no micro-organisms and resolved spontaneously with no dietary modifications. However he was failing to thrive. At $7 \frac{1}{2}$ months he developed type I diabetes mellitus, which was difficult to control. At this point a possible diagnosis of autoimmune polyglandular endocrinopathy, candidiasis and ectodermal dysplasia (APECED) was considered. Screening for other endocrine dysfunctions e.g: thyroid, adrenal was negative. An episode of self-resolving Coomb's positive autoimmune haemolytic anaemia complicated the disease course at 18 months. The possibility of IPEX syndrome was considered but confirmatory testing was unavailable.

At $3 \frac{1}{2}$ years he developed biopsy-proven membranoproliferative nephrotic syndrome with hypertension. It responded to a course of steroids. FOXP3 genetic testing at this time confirmed the diagnosis of IPEX syndrome. He is now $4 \frac{1}{2}$ years old with a developmental age of $3 \frac{1}{2}$ years and is on insulin treatment for diabetes. Routine immunisations including live vaccines for polio, measles and rubella have had no untoward effects to date.

\section{Discussion}

This boy's clinical course not only illustrates the natural history of the condition but also possible pitfalls in diagnosis in a setting of inadequate investigative facilities. Nephrotic syndrome due to membrano-proliferative glomerulonephritis has not been reported previously with IPEX syndrome.

The incidence of IPEX syndrome is unknown. It is an autoimmune condition with dysfunction of T-cells due to mutations in the fork-head box P3 (FOXP3) gene [1]. Over 35 mutations of this gene have been described. Analysis of 57 patients IPEX syndrome showed that diarrhoea, type I diabetes and dermatitis were the commonest presentations [2]. Failure to thrive, increased susceptibility to infection, anaemia and thrombocytopaenia are other features. Most children succumb to the disease within 2 years. Our patient presented with candidiasis, an indication of T-cell dysfunction, followed by classical features.

Adequate glycaemic control has proven to be a continuous challenge in his management and is a known problem in patients with IPEX syndrome [2]. Diarrhoea was not a marked feature in our patient although IPEX syndrome is usually associated with prominent enteropathic features which do not abate spontaneously [2]. Nephrotic syndrome with minimal change or membranous changes and interstitial nephritis have been reported in IPEX syndrome [3,4]. Our patient however had membranoproliferative glomerulonephritis. He responded to a course of steroids and has subsequently remained in remission without immunosuppression.

Confirmatory diagnosis was possible only when the child was $3 \frac{1}{2}$ years of age when the in-frame deletion of FOXP3 at Exon 8 (c.751_753del) resulting in the protein p.Glu251del. was identified with his mother being heterozygous. This particular deletion has not been reported from a developing country previously.

Medical management of IPEX syndrome involves addressing individual issues and also attempts at controlling the overwhelming autoimmune activity by use of immunusuppressants such as sirolimus. The only form of cure has been allogenic stem cell transplantation [5]. The cost of this procedure is currently prohibitive in the

${ }^{1}$ University Paediatric Unit, Lady Ridgeway Hospital for Children, ${ }^{2}$ Department of Paediatrics, University of Colombo, Sri Lanka.

Correspondence: RR, e-mail: <ranmali_waduge@yahoo.com>. Received 28 May and revised version accepted 11 October 2012. Competing interests: none declared. 
case of our patient. Most children with IPEX syndrome do not survive beyond their second birthday but those with milder phenotypes who have survived longer have had prominent enteropathic features and less problems with diabetes which is the converse of what is seen in our patient. Our patient's mutation has been described previously, but does not have a known association with milder clinical features or nephrotic syndrome of a particular type [1].

\section{Acknowledgements}

We thank Dr. D Muthukuda and Royal Devon and Exeter Hospital for molecular genetic analysis.

\section{References}

1. Chatila TA, Blaesea F, Ho N, et al. JM2, encoding a fork head-related protein, is mutated in X-linked autoimmunity- allergic disregulation syndrome. Journal of Clinical Investigation 2000; 106: R75-81.

2. Wildin RS, Smyk-Pearson S, Filipovich AH. Clinical and molecular features of the immunodysregulation, polyendocrinopathy, enteropathy, X linked (IPEX) syndrome. Journal of Medical Genetics 2002; 39: 537-45.

3. Moudgil A, Perriello P, Loechelt B, et al. Immunodysregulation, polyendocrinopathy, enteropathy, X-linked (IPEX) syndrome: an unusual cause of proteinuria in infancy. Pediatric Nephrology 2007; 22: 1799-802.

4. Hashimura Y, Nozu K, Kanegane H, et al. Minimal change nephrotic syndrome associated with immune dysregulation, polyendocrinopathy, enteropathy, X-linked syndrome. Pediatric Nephrology 2009; 24: 1181-6.

5. Lucas K, Ungar D, Comito M, et al. Submyeloablative cord blood transplantation corrects clinical defects seen in IPEX syndrome. Bone Marrow Transplantation 2007; 39: 55-6. 